\title{
Densidad de microorganismos en rizósfera de limón mexicano con HuangLongBing e inoculado con hongos micorrízicos arbusculares y bacterias benéficas en invernadero Microorganism density in the rhizosphere of Mexican lime with HuangLongBing and inoculated with arbuscular mycorrhizal fungi and beneficial bacteria in greenhouse
}

\author{
Christian Salvador Mendoza-Hernández ${ }^{1,2}{ }^{(-)}$, Evangelina Esmeralda Quiñones-Aguilar ${ }^{2}{ }^{(}$, \\ Guadalupe Esperanza López-Ramírez ${ }^{1,2}$, Luis López-Pérez ${ }^{3}$ y Gabriel Rincón-Enríquez $^{2, *}$
}

\footnotetext{
${ }^{1}$ Ingeniería en Agrotecnología, Universidad Politécnica del Bicentenario. Carretera libre Silao-Romita km 2, Col. San Juan de los Durán. 36283 Silao de la Victoria, Guanajuato, México.

${ }^{2}$ Laboratorio de Fitopatología, Biotecnología Vegetal, Centro de Investigación y Asistencia Tecnológica y Diseño del Estado de Jalisco A.C. Camino Arenero 1227, Col. El Bajío del Arenal. 45019 Zapopan, Jalisco, México.

\$ Autor para correspondencia (grincon@ciatej.mx)

${ }^{3}$ Instituto de Investigaciones Agropecuarias y Forestales, Universidad Michoacana de San Nicolás de Hidalgo. Carretera Morelia-Zinapécuaro km 9.5. 58880 Tarímbaro, Michoacán, México.
}

\section{RESUMEN}

México está entre los primeros productores de limón mexicano (Lm), con 2.5 millones de hectáreas por año. Actualmente esta actividad agrícola se ve amenazada por el HuangLonBing (HLB), causada por la bacteria Candidatus Liberibacter asiaticus (CLas). El desarrollo de estrategias biotecnológicas dirigidas al combate de esta enfermedad es indispensable para incorporarlas al manejo actual. El objetivo del presente trabajo consistió en aislar y determinar la abundancia de bacterias (B) y hongos filamentosos (HF) totales de rizosfera de plantas de Lm infectadas con CLas y tratadas con Bacillus subtilis (BS) o Funneliformis mosseae (FM). Se empleó un diseño experimental completamente al azar con seis tratamientos con cinco repeticiones con árboles de un año y medio e infectados con CLas vía injerto de yema siendo estos: 1) aplicación mensual de BS $\left(5 \times 10^{5}\right.$ UFC $\mathrm{g}^{-1}$ de sustrato: BsCLas); 2) aplicación única de FM (5 esporas $\mathrm{g}^{-1}$ inóculo: FmCLas); 3) fertilización química sin CLas (Q); 4) fertilización química con CLas (QCLas); 5) fertilización orgánica sin CLas (O) y 6) fertilización orgánica con CLas (OCLas). Se realizaron cinco muestreos rizosféricos, cada 70 días

Cita recomendada:

Mendoza-Hernández, C. S., E. E. Quiñones-Aguilar, G. E. López-Ramírez, L. López-Pérez y G. Rincón-Enríquez. 2019. Densidad de microorganismos en rizósfera de limón mexicano con HuangLongBing e inoculado con hongos micorrízicos arbusculares y bacterias benéficas en invernadero. Terra Latinoamericana 37: 379-389.

DOI: https://doi.org/10.28940/terra.v37i4.525 después de la primera aplicación (ddpa) de la nutrición química y orgánica o después de la inoculación de los microorganismos. Las poblaciones de $\mathrm{B}$ y $\mathrm{HF}$ se determinaron mediante la técnica de diluciones decimales, siembra en placa (NA y PDA) y conteo de UFC. Se encontraron diferencias estadísticas (Tukey, $P \leq 0.05)$ en la dinámica de $\mathrm{B}$ y $\mathrm{HF}$ en tratamientos con $C$ Las con respecto a sin infectar. A los 368 ddpa en B totales se encontraron $7.4 \times 10^{5} \mathrm{UFC}^{-1}$ de sustrato en QCLas en contraste con las $2.67 \times 10^{6} \mathrm{UFC} \mathrm{g}^{-1} \mathrm{de}$ sustrato para $\mathrm{Q}$. La dinámica de $\mathrm{B}$ fue mayormente afectada en comparación con HF. La inoculación de microorganismos benéficos o aplicación de nutrición orgánica fueron incapaces de mantener las poblaciones de B totales.

Palabras clave: Bacillus subtilis, citricos, Candidatus Liberibacter asiaticus, Funneliformis mosseae.

\section{SUMMARY}

Mexico is among the first producers of Mexican lime (M1), with 2.5 million hectares per year. Currently, this agricultural activity is threatened by HuangLonBing

Recibido: 14 de marzo de 2019 .

Aceptado: 31 de mayo de 2019.

Publicado en Terra Latinoamericana 37: 379-389. 
(HLB), caused by the bacterium Candidatus Liberibacter asiaticus (CLas). The development of biotechnological strategies aimed at combating this disease is imperative in order to incorporate them into the current management. The objective of our study was to isolate and determine the abundance of total bacteria (B) and filamentous fungi (FF) in the rhizosphere of Ml plants infected with CLas, treated with Bacillus subtilis (BS) and Funneliformis mosseae (FM). A completely randomized experimental design was used in six treatments and five replicates with one and a half year old trees infected with $C$ Las via bud grafting as follows: 1) monthly application of BS $(5 \times$ $10^{5} \mathrm{CFU} \mathrm{g}^{-1}$ of substrate: BsCLas), 2) single application of FM (5 spores $\mathrm{g}^{-1}$ inoculum: FmCLas); 3) chemical fertilization without $C$ Las $(\mathrm{Q}), 4)$ chemical fertilization with CLas (QCLas); 5) organic fertilization without CLas (O) and 6) organic fertilization with CLas (OCLas). Five rhizospheric samplings were carried out each 70 days after the first application (dafa) of the chemical and organic nutrition of after the inoculation of microorganisms. The populations of bacteria (B) and fungi (FF) were determined using the decimal dilution method, plating (AN and PDA) and CFU count. Statistical differences (Tukey's, $P \leq 0.05$ ) were found in the population dynamics of $\mathrm{B}$ and total FF in treatments infected with CLas, with regard to noninfected treatments. At 368 dafa, total substrate B were $7.4 \times 10^{5} \mathrm{CFU} \mathrm{g}^{-1}$ in treatment QCLas, in contrast, total substrate B were $2.67 \times 10^{6} \mathrm{CFU} \mathrm{g}^{-1}$ in treatment Q. The population dynamics of B was the most affected compared to that of FF. The inoculation of beneficial microorganisms or application of organic nutrition were unable to maintain total B populations.

Index words: Bacillus subtilis, citrus, Candidatus Liberibacter asiaticus, Funneliformis mosseae.

\section{INTRODUCCIÓN}

México es el cuarto país productor de cítricos en el mundo (Miranda-Salcedo et al., 2012'1) y segundo lugar de limón. El cultivo de limón mexicano (Lm) se desarrolla principalmente en las costas del pacífico mexicano (González-Sánchez y Echevarria, 2003), con una producción que superó las 2.5 millones de toneladas para 2017 (SIAP, 2019). En la actualidad esta actividad agrícola se ve amenazada por la enfermedad denominada HLB (Sánchez-Borja et al., 2010), cuyo agente causal es Candidatus Liberibacter asiaticus (CLas) (Kim et al., 2008). CLas es una bacteria fastidiosa, Gram negativa y cuyo hábitat es el floema presentando una distribución irregular en el árbol (Robles et al., 2010). Su sintomatología se caracteriza por el desarrollo de un ataque sectorial, apareciendo en una sola rama y las hojas presentan manchas cloróticas difusas (jaspeado), posteriormente estas se amarillentan y caen. Finalmente, los árboles mueren al cabo de algunos años (Armenta-Cardenas et al., 2010).

El estado de nutrición de las plantas genera resistencia a las enfermedades, la cual influye en la comunidad microbiológica de la rizosfera, que en consecuencia contribuye al cambio de la composición del floema induciendo resistencia sistémica a bacterias u hongos endófitos (Shen et al., 2013). El estado nutrimental de las plantas puede ser afectado químicamente por productos agrícolas aplicados a ellas de manera artificial o por la utilización de microorganismos benéficos (Santillano et al., 2019). Referente a esta última, se encuentran los hongos micorrízicos arbusculares (HMA). Los HMA fueron descritos por primera vez a principios de la década de 1880, cuando el patólogo forestal alemán A. B. Frank, acuñó el término mykorhiza, haciendo referencia a la interacción entre una especie de hongo y la raíz de una especie vegetal (Andrade-Torres, 2010). En esta interacción, la planta le proporciona carbohidratos (azúcares, producto de su fotosíntesis) y un microhábitat al hongo para completar su ciclo de vida; el hongo le proporciona a la planta una mejor captación de agua, minerales de baja disponibilidad (P, $\mathrm{N}, \mathrm{K}, \mathrm{Ca}, \mathrm{Mg}, \mathrm{Fe}, \mathrm{Zn}$ y Mn) e induce la defensa contra patógenos (Sarabia et al., 2010; Camargo-Ricalde et al., 2012; Trinidad et al., 2017). Los HMA generan una respuesta diferente dependiendo de la planta hospedera y no son específicos a una especie o a un grupo vegetal en particular (Hernández-Cuevas et al., 2003), no pueden desarrollar su ciclo sin un hospedero (son obligados), y crecen solo en raíces de plantas hospederas (Hernández-Cuevas et al., 2003). Por otra parte, también se encuentran las bacterias promotoras de crecimiento vegetal (BPCV), las cuales pueden

${ }^{1}$ Miranda-Salcedo, M., J. López Arroyo, J. Padrón Chávez y J. Velázquez Monreal. 2012. Fitosanidad y problemática de la citricultura en el valle de Apatzingán, Michoacán. In: Congreso Mexicano de Investigación en Cítricos 2012. Centro de Investigación Regional Golfo-Centro (CIRGOC), Campo Experimental Ixtacuaco. Veracruz México. 
incrementar el crecimiento y productividad vegetal sin dañar o contaminar el suelo (Loredo-Osti et al., 2004). Las BPCV pueden solubilizar fosfatos, producir hormonas o fijar nitrógeno, lo que afecta directamente a las plantas de una forma benéfica, incrementando el fluido del agua y minerales, mejorando el desarrollo radicular y aumentando la actividad enzimática de la planta; incluso algunas tienen la capacidad de control bilógico, las cuales promueven el crecimiento de la planta al suprimir los fitopatógenos que la atacan (DeBashan et al., 2007). Entre las BPCV más conocidas están las especies que pertenecen a los géneros Rhizobium, Pseudomonas, Azospirillum y Bacillus. El análisis de las poblaciones de microorganismos de la rizósfera puede reflejar las condiciones y la diversidad microbiana encontrada, así como la salud de un ecosistema; sin embargo, existen varios factores que pueden alterar las poblaciones de microorganismos tal es el caso de la temperatura, radiación ultravioleta, radiación ionizante, humedad, $\mathrm{pH}$, potencial REDOX, ácidos orgánicos, sales y gases tales como nitrógeno y oxígeno (Calvo et al., 2008). No existen trabajos previos sobre la dinámica poblacional de bacterias y hongos de la rizósfera de plantas de Lm infectadas por $C$ Las. Su estudio puede abrir un panorama sobre el control y manejo de la enfermedad relacionada a este fitopatógeno. El objetivo del trabajo fue aislar y determinar la abundancia de bacterias (B) y hongos filamentosos (HF) totales en muestras de sustrato de plantas de limón infectadas con CLas y tratadas con Bacillus subtilis (BS) o Funneliformis mosseae (FM).

\section{MATERIALES Y MÉTODOS}

El experimento tuvo una duración de un año bajo condiciones de invernadero en las instalaciones de la Unidad Zapopan del CIATEJ (20.700861, -103.473855). Consistió en un diseño completamente al azar con seis tratamientos utilizando árboles certificados de Citrus aurantifolia injertados sobre Citrus macrophylla de un año y medio, provenientes de Tecomán Colima en bolsas de $2 \mathrm{~kg}$ de sustrato. Los árboles de limón mexicano $(\mathrm{Lm})$ se infectaron con Candidatus Liberibacter asiaticus (Clas) vía injerto de yema, utilizando varetas de árboles sintomáticos provenientes de huertos de Lm de Tecomán Colima, el injerto se hizo ocho meses previo al trasplante a macetas de $20 \mathrm{~L}$ y al comienzo del experimento. Los árboles se regaron a capacidad de campo por lo menos una vez por semana. La descripción de los tratamientos es mostrada en el Cuadro 1, al momento del trasplante se aplicó Funneliformis mosseae a la raíz de los árboles. La primera aplicación de tratamientos con B. subtilis, nutrición química y nutrición orgánica se inició a los 30 días posteriores de la inoculación con $F$. mosseae. A los tratamientos que recibieron nutrición química (Q) se les aplicó urea (46\% de N) y triple 16 (16-16-16\% de N-P-K) cada tres meses (cuatro veces) sumando una dosis total de 150-18-18 (12.8 g de urea más $2.6 \mathrm{~g}$ de triple 16 por unidad experimental). A los dos tratamientos que recibieron nutrición orgánica $(\mathrm{O})$, se les suministró lombricomposta líquida de manera mensual (Cuadro 1). El sustrato empleado en el experimento fue

Cuadro 1. Diseño de tratamientos para evaluar el efecto de aplicación de microrganismos benéficos en las poblaciones de bacterias y hongos de la rizosfera de plantas de limón mexicano enfermo con HLB.

Table 1. Design of treatments performed to evaluate the effect of the application of beneficial microorganisms over populations of bacteria and fungi from the rhizosphere of Mexican lime plants infected with HLB.

Tratamiento

Condiciones de aplicación

\begin{tabular}{llllll}
\hline Clave & Descripción & CLas & Concentración & Total & Periodicidad \\
\hline FmCLas & Funneliformis mosseae+FQ & Con & 5 esporas g inóculo $^{-1}$ & $100 \mathrm{~g}$ & Inicio \\
BsCLas & Bacillus subtilis+FQ & Con & $2.5 \times 10^{8} \mathrm{UFC} \mathrm{mL}^{-1}$ & $100 \mathrm{~mL}$ & Cada 30 días \\
QCLas & Fertilización química $\left(\mathrm{kg} \mathrm{ha}^{-1}\right)$ & Con & $37.5-4.5-4.5$ & $150-18-18$ & Cada 90 días \\
Q & Fertilización química $\left(\mathrm{kg} \mathrm{ha}^{-1}\right)$ & Sin & $37.5-4.5-4.5$ & $150-18-18$ & Cada 90 días \\
OCLas & Fertilización orgánica & Con & $47000 \mathrm{mg} \mathrm{kg}^{-1}$ & $100 \mathrm{~mL}$ & Cada 30 días \\
O & Fertilización orgánica & Sin & $47000 \mathrm{mg} \mathrm{kg}^{-1}$ & $100 \mathrm{~mL}$ & Cada 30 días \\
\hline
\end{tabular}

$\overline{\mathrm{FQ}}=$ fertilización química: urea $(46 \% \mathrm{~N})$, triple 16. Fertilización orgánica $=$ lombricomposta líquida. CLas= Candidatus Liberibacter asiaticus. $\mathrm{FQ}=$ chemical fertilization: urea $(46 \% \mathrm{~N})$, triple 16. Organic fertilization $=$ liquid vermicompost. $C$ Las $=$ Candidatus Liberibacter asiaticus. 
una mezcla suelo:arena:agrolita:turba (Sunshine Mix $^{\circledR}$ No. 3) (50:30:10:10, v/v/v/v). Previo a la esterilización del sustrato en autoclave $\left(120{ }^{\circ} \mathrm{C}, 1.05 \mathrm{~kg} \mathrm{~cm}^{-2}, 6 \mathrm{~h}\right)$ se realizó el conteo de B y HF totales. Se emplearon recipientes de plástico negro con capacidad de $20 \mathrm{~L}$ como macetas para cada planta de limón, en cada maceta se colocaron $16 \mathrm{~kg}$ de la mezcla esterilizada. La mezcla esterilizada presentó una textura franca, $\mathrm{pH}$ neutro en agua (6.52), contenido de materia orgánica de $13.5 \%$, conductividad eléctrica de $3.31 \mathrm{dS} \mathrm{m}^{-1}$, contenido de N, P, K, Ca y Mg de 51, 184, 445, 3348 y $712 \mathrm{mg} \mathrm{kg}^{-1}$ respectivamente, estos análisis fueron realizados por el Laboratorio de Análisis Agrícola de la empresa Fertilab ${ }^{\circledR}$ de Celaya Guanajuato (fertilab.com. $\mathrm{mx}$ ) de acuerdo a los procedimientos descritos en la Norma Oficial Mexicana (NOM-021-RECNAT-2000, 2002). Posteriormente de la aplicación de cada uno de los tratamientos se realizaron cuatro muestreos rizosféricos a 70, 140, 210 y 280 días después de la primera aplicación (ddpa) así como un muestreo al final del experimento (368 ddpa), utilizando un tubo de PVC de $1.27 \mathrm{~cm}$ de diámetro y $35 \mathrm{~cm}$ de alto, introduciéndolo en cuatro puntos alrededor de la planta (Norte, Sur, Este y Oeste) a tres diferentes profundidades $(5,15$ y $30 \mathrm{~cm})$, posteriormente éstas muestras fueron homogenizadas y se almacenaron a $4{ }^{\circ} \mathrm{C}$ hasta su procesamiento. Se analizaron cinco repeticiones por tratamiento. Una unidad experimental consistió en una maceta con un árbol de limón mexicano. Para el conteo de bacterias totales se utilizó el medio de cultivo agar nutritivo (AN, 213000 DIFCO $^{\circledR}$ ), mientras para la determinación de hongos totales se empleó el medio agar papa dextrosa (PDA, 213400 DIFCO $^{\circledR}$ ), a pH de 5.5, $3.3 \mathrm{~mL} \mathrm{~L}^{-1}$ de rosa de bengala al $1 \%\left(198250 \mathrm{SIGMA}^{\circledR}\right)$ y $40 \mu \mathrm{g} \mathrm{mL}^{-1}$ de estreptomicina $\left(\right.$ S9137, SIGMA $\left.^{\circledR}\right)$. La cuantificación de las unidades formadoras de colonia (UFC) se realizó por medio de diluciones decimales seriadas en cada muestra siguiendo lo propuesto por Camacho et al. (2009), con algunas modificaciones. Para lo cual se determinó el peso seco de las muestras a analizar, posteriormente para cada uno de los tratamientos se tomaron $10 \mathrm{~g}$ de muestra y se le añadieron $90 \mathrm{~mL}$ de agua destilada para obtener la dilución $10^{-1}$, prosiguiendo a tomar $1 \mathrm{~mL}$ de la dilución $10^{-1}$ y disolverla en $9 \mathrm{~mL}$ de agua destilada para obtener la dilución $10^{-2}$. Repitiendo sucesivamente los pasos mencionados anteriormente hasta la dilución $10^{-5}$.

Los datos obtenidos se procesaron mediante un análisis de varianza de una vía y mediante la comparación múltiple de medias de Tukey, ambos a un nivel de significancia del 5\% $(P \leq 0.05)$ mediante el programa estadístico Statgraphics ${ }^{\circledR}$ Centurion XV versión 15.2.06 (StatPoint Inc. 2005). Los resultados obtenidos a partir del análisis estadístico se presentan de la manera siguiente: 1) el efecto producido en las poblaciones de $\mathrm{B}$ y $\mathrm{HF}$ totales, por la inoculación de F. mosseae (FmCLas) y B. subtilis (BsCLas) en contraste con el tratamiento QCLas dado que los tratamientos FmCLas y BsCLas tuvieron fertilización química; 2) el comparativo poblacional de B y HF totales, en los tratamientos QCLas y Q; y 3) el contraste poblacional de $\mathrm{B}$ y HF totales obtenido de los tratamientos OCLas y O con respecto a los tratamientos QCLas y Q con el fin de determinar el efecto de distintas formas de fertilización. Con este análisis se comprende el fenómeno biológico ocurrido al aplicar los microorganismos benéficos, así como la nutrición orgánica contra la química en las plantas de $\mathrm{Lm}$ infectadas con CLas.

Para determinar la presencia de CLas en los árboles de limón inoculados con el patógeno se realizó una PCR punto final. La extracción de ADN de realizó a partir de las nervaduras centrales de las hojas utilizando el protocolo CTAB (cetyltrimethylammonium bromide) a 3\% de acuerdo a Zhang et al. (1998). Para la reacción PCR punto final, se amplificó la secuencia parcial del gen ribosomal $16 \mathrm{~S} \mathrm{ADNr}$ (1160 pb) con los oligonucleótidos específicos OI1 (5'-GCGCGTATGCAATACGAGCGGCA- 3') y OI2c (5'-GCCTCGCGACTTCGCAACCCAT-3') (Jagoueix et al., 1994), empleando las siguientes condiciones: $94{ }^{\circ} \mathrm{C}$ por $5 \mathrm{~min} ; 35$ ciclos de $45 \mathrm{~s}$ a $94{ }^{\circ} \mathrm{C}, 45 \mathrm{~s}$ a $68{ }^{\circ} \mathrm{C}, 60 \mathrm{~s}$ a $72{ }^{\circ} \mathrm{C}$; un ciclo final a $72{ }^{\circ} \mathrm{C}$ por $10 \mathrm{~min}$ (Lou et al., 2012). La reacción de PCR se migró por electroforesis ( $90 \mathrm{~V}$ por $40 \mathrm{~min}$ ) en geles de agarosa a $0.8 \%$ y se observó en un transiluminador con GelRed ${ }^{\circledR}$ (1X).

\section{RESULTADOS Y DISCUSIÓN}

\section{Infección de los Árboles de Limón Mexicano con el Agente Causal del HLB (CLas)}

Las plantas de limón que fueron infectadas con Candidatus Liberibacter asiaticus (Clas) por medio del injerto de yema se enfermaron con HLB. La extracción de ADN fue efectiva utilizando el método de CTAB (Zhang et al., 1998) (Figura 1A) y se amplificó la secuencia parcial del gen ribosomal $16 \mathrm{~S} \mathrm{ADNr}$ 
de la bacteria CLas (1167 pb) con los oligonucleótidos específicos OI1 y OI2c (Lou et al., 2012) (Figura 1B). Esto demuestra que las plantas de limón fueron infectadas con $C$ Las a través del injerto de yema.

\section{Efecto de la Aplicación de Microorganismos Benéficos en las Poblaciones de Bacterias y Hongos Filamentosos Totales en Plantas de Limón Infectadas con $C$ Las}

Las poblaciones de microorganismos están altamente influenciadas por los exudados radicales que incluyen aminoácidos, ácidos grasos, nucleótidos, ácidos orgánicos, fenoles, reguladores de crecimiento, poliamidas, esteroles, azúcares y vitaminas (Wang et al., 2018; Ramírez et al., 2013), los cuales pueden ser modificados a través de la interacción de las plantas con los microorganismos promotores de crecimiento vegetal (González y Fuentes, 2017).

Al sustrato inicial se le determinó el número de $\mathrm{UFC} \mathrm{g}^{-1}$ de sustrato de bacterias y hongos filamentosos totales, constatando su presencia previa al experimento, presentando $1.56 \times 10^{5} \mathrm{UFC} \mathrm{g}^{-1}$ de sustrato de bacterias

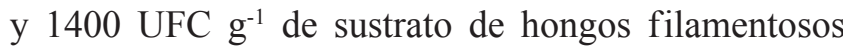
totales.

En tres de los cinco muestreos los conteos de UFC de bacterias totales para el tratamiento QCLas fueron inferiores con respecto a los tratamientos inoculados con los microorganismos benéficos (Cuadro 2). La inoculación de F. mosseae (FmCLas) y B. subtilis (BsCLas) influye positivamente en la población de los microorganismos de la rizosfera (Cuadro 2), mostrando diferencias significativas en comparación con el tratamiento infectado (QCLas).
A)

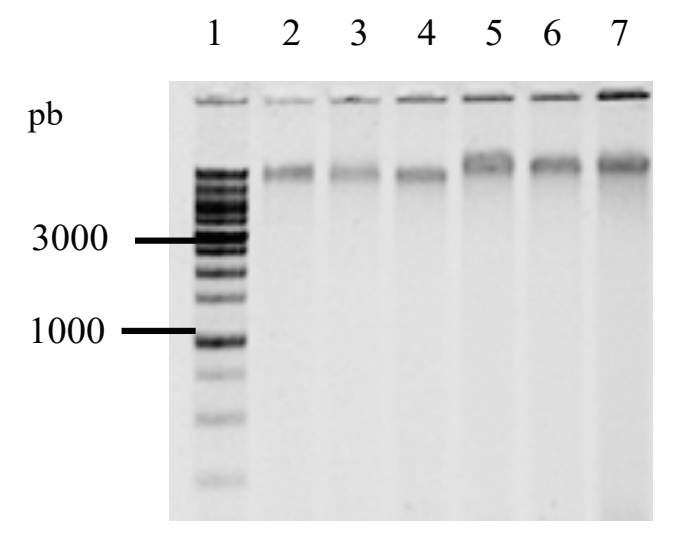

B)

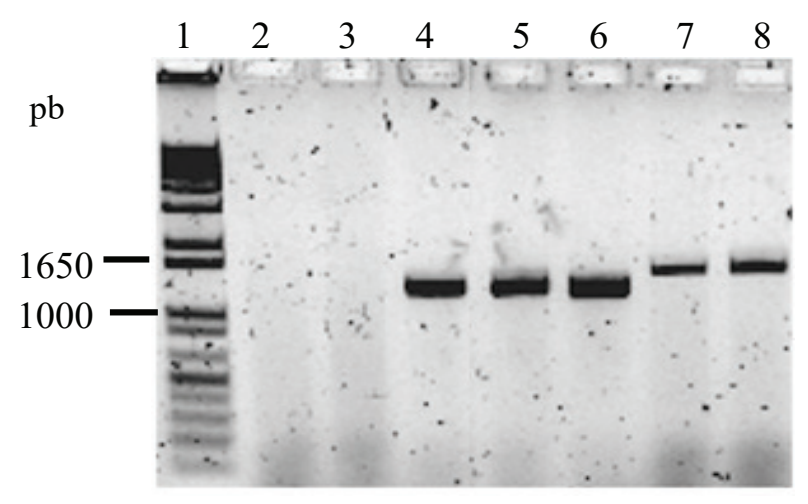

Figura 1. Determinación de la presencia de Candidatus Liberibacter asiaticus (Clas) en plantas de limón mexicano inoculadas con la bacteria fitopatógena Candidatus. A) Extracción de ADN de plantas de limón mexicano, utilizando el protocolo de CTAB a 3\%. Carril 1: $1 \mathrm{~Kb}$ Leadder Plus (Invitrogen); carril de 2 al 7: ADN de plantas de limón con y sin inoculación de CLas. B) Determinación de la presencia de CLas en árboles de limón mediante PCR punto final (1167 pb) de plantas injertadas con varetas de árboles enfermos con HLB. Carril 1: $1 \mathrm{~Kb}$ Leadder Plus (Invitrogen); carril 2: plantas no inoculadas con CLas y con tratamiento de fertilización química (Q); carril 3: plantas no inoculadas con $C$ Las y con tratamiento de fertilización orgánica $(\mathrm{O})$; carril 4: plantas inoculadas con CLas y con tratamiento de fertilización química (QCLas); carril 5: plantas inoculadas con CLas y con tratamiento de fertilización orgánica (OCLas); carril 6: plantas inoculadas con CLas y con inoculación de Bacillus subtilis (BsCLas); carril 7: plantas inoculadas con CLas y con inoculación de Funneliformis mosseae (FmCLas); carril 8: plásmido con ADN de CLas.

Figure 1. Determination of Candidatus Liberibacter asiaticus (CLas) in Mexican lime plants inoculated with the phytopathogenic bacterium Candidatus. A) DNA extraction from Mexican lime plants using the protocol CTAB. Lane 1: $1 \mathrm{~Kb}$ Ladder Plus (Invitrogen); lane 2 to 7: DNA from Mexican lime with and without inoculation of $C$ Las. B) Determination of CLas in Mexican lime trees by endpoint PCR (1167 pb) of plants grafted with spikes of trees infected with HLB. Lane 1: $1 \mathrm{~Kb}$ Ladder Plus (Invitrogen); lane 2: plants without inoculation with CLas and treated with chemical fertilization (Q); lane 3: plants without inoculation with $C$ Las and treated with organic fertilization $(\mathrm{O})$; lane 4: plants inoculated with $C$ Las and treated with chemical fertilization (QCLas); lane 5: plants inoculated with CLas and treated with organic fertilization (OCLas); lane 6: plants inoculated with CLas and Bacillus subtilis (BsCLas); lane 7: plants inoculated with CLas and Funneliformis mosseae (FmCLas); lane 8: plasmid with CLas DNA. 
Cuadro 2. Dinámica poblacional de bacterias totales después de la primera aplicación de microorganismos benéficos en árboles de limón mexicano bajo condiciones de invernadero.

Table 2. Population dynamics of total bacteria after the first application of beneficial microorganisms in Mexican lime trees under greenhouse conditions.

\begin{tabular}{lccccc}
\hline & \multicolumn{5}{c}{ Días después de la primera aplicación $\left(10^{6} \mathrm{UFC} \mathrm{g}^{-1}\right.$ sustrato $)$} \\
\cline { 2 - 6 } Tratamiento & 70 & 140 & 210 & 280 & 368 \\
\hline FmCLas & $5.37 \mathrm{c}$ & $5.00 \mathrm{a}$ & $2.50 \mathrm{~b}$ & $0.76 \mathrm{~b}$ & $0.46 \mathrm{a}$ \\
BsCLas & $3.40 \mathrm{~b}$ & $4.83 \mathrm{a}$ & $3.00 \mathrm{~d}$ & $0.62 \mathrm{~b}$ & $0.65 \mathrm{ab}$ \\
QCLas & $0.56 \mathrm{a}$ & $13.50 \mathrm{~b}$ & $0.45 \mathrm{a}$ & $0.37 \mathrm{a}$ & $0.74 \mathrm{~b}$ \\
Q & $0.55 \mathrm{a}$ & $6.87 \mathrm{a}$ & $2.63 \mathrm{c}$ & $0.65 \mathrm{~b}$ & $2.67 \mathrm{c}$ \\
\hline
\end{tabular}

Letras distintas en la misma columna indican diferencias significativas, según la prueba de Tukey $(P \leq 0.05)$.

Different letters in the same column indicate significant differences according to the Tukey's test $(P \leq 0.05)$.

Todos los muestreos presentaron un incremento en la población de bacterias en comparación con el conteo inicial $\left(1.56 \times 10^{5} \mathrm{UFC} \mathrm{g}^{-1}\right.$ de sustrato).

La inoculación con F. mosseae a los 70 y 140 ddpa presentó su mayor incremento en la población de bacterias totales $\left(5.37 \times 10^{6}\right.$ y $5 \times 10^{6} \mathrm{UFC} \mathrm{g}^{-1} \mathrm{de}$ sustrato respectivamente; Cuadro 2), en contraste con el conteo inicial $\left(1.56 \times 10^{5} \mathrm{UFC} \mathrm{g}^{-1}\right.$ de sustrato $)$. Con respecto al aumento del conteo de UFC, Gui et al. (2017) mencionan que los HMA pueden impactar en las comunidades bacterianas de la rizósfera, produciendo nichos para su crecimiento, además de mejorar la actividad de éstas asociándolo con la disponibilidad de N. Posteriormente, la población de bacterias totales disminuyó a partir de los 280 ddpa hasta su mínimo conteo de $4.6 \times 10^{5} \mathrm{UFC} \mathrm{g}^{-1}$ de sustrato a los 368 ddpa (Cuadro 2), el cual fue además estadísticamente diferente $(P \leq 0.05)$ al tratamiento QCLas. Cui et al. (2018), mencionan que los HMA además de participar en la regulación de las defensas de las plantas pueden también mantener la estabilidad del microecosistema del suelo. Gui et al. (2017), reportan aumento en la abundancia de los filos Actinobacteria y Thermoleophilia, así como un aumento en las etapas tempranas de Alphaproteobacterias para posteriormente observar una disminución de estas mismas en muestras de sustrato forestal inoculadas con F. mosseae. Reportando por otra parte la disminución de la abundancia de Acidobacterias y Gammaproteobacterias, en todos los muestreos realizados.

La inoculación con $B$. subtilis presentó una dinámica poblacional similar al tratamiento inoculado con el HMA, con conteos de 3.40, 4.83 y $3 \times 10^{6} \mathrm{UFC} \mathrm{g}^{-1}$ de sustrato a los 70, 140 y 210 ddpa respectivamente. Denotando una disminución a los 280 y 368 ddpa (Cuadro 2). El aumento en el conteo de UFC en los tres primeros muestreos fue un efecto esperado, ya que B. subtilis se inoculó de manera mensual (Cuadro 2). Con respecto a la disminución de la población subsecuente, se ha reportado que $B$. subtilis tiene una excelente habilidad de colonización radicular, produce antibiosis, lisis celular, competición por espacio y nutrientes (Wang et al., 2018), generando competencia con las bacterias de la rizósfera.

La dinámica de población de hongos filamentosos totales (Cuadro 3) mostró un comportamiento similar en los tres tratamientos infectados con CLas. La inoculación de $F$. mosseae y $B$. subtilis generó una estabilidad en el conteo de UFC de hongos filamentosos, manteniendo el conteo de UFC entre un intervalo

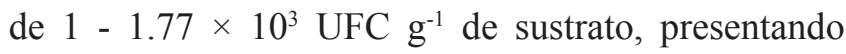
solamente en un muestreo diferencias estadísticas (Tukey, $P \leq 0.05$ ) (280 ddpa). El tratamiento infectado con CLas (CLasQ) mostró un comportamiento similar a los tratamientos inoculados con los microorganismos benéficos. Siendo en la mayoría de los tratamientos estadísticamente iguales (Cuadro 3).

\section{Efecto de CLas en las Poblaciones de Bacterias y Hongos Filamentosos Totales en Árboles de Limón}

En contraste con las plantas que crecen en condiciones de asepsia en el laboratorio, las plantas sanas en la naturaleza, forman asociaciones con comunidades microbianas benéficas o incluso interacciones con microorganismos fitopatógenos pertenecientes a diversos reinos (Hacquard et al., 2017). Para conocer 
Cuadro 3. Dinámica poblacional de hongos filamentosos totales después de la primera aplicación de microorganismos benéficos en árboles de limón mexicano bajo condiciones de invernadero.

Table 3. Population dynamics of total filamentous fungi after the first application of beneficial microorganisms in Mexican lime trees under greenhouse conditions.

\begin{tabular}{lccccc}
\hline \multirow{2}{*}{ Tratamiento } & \multicolumn{5}{c}{ Días después de la primera aplicación $\left(10^{3} \mathrm{UFC}^{-1}\right.$ sustrato $)$} \\
\cline { 2 - 5 } & 70 & 140 & 210 & 280 & 368 \\
\hline FmCLas & $1.27 \mathrm{a}$ & $1.00 \mathrm{a}$ & $1.47 \mathrm{a}$ & $1.03 \mathrm{a}$ & $1.33 \mathrm{a}$ \\
BsCLas & $1.63 \mathrm{a}$ & $1.00 \mathrm{a}$ & $1.07 \mathrm{a}$ & $1.77 \mathrm{~b}$ & $1.77 \mathrm{a}$ \\
QCLas & $1.87 \mathrm{a}$ & $3.67 \mathrm{~b}$ & $1.00 \mathrm{a}$ & $1.77 \mathrm{~b}$ & $3.93 \mathrm{ab}$ \\
Q & $2.13 \mathrm{a}$ & $1.50 \mathrm{a}$ & $1.03 \mathrm{a}$ & $1.83 \mathrm{~b}$ & $5.27 \mathrm{~b}$ \\
\hline
\end{tabular}

Letras distintas en la misma columna indican diferencias significativas, según la prueba de Tukey $(P \leq 0.05)$.

Different letters in the same column indicate significant differences, according to the Tukey's test $(P \leq 0.05)$.

como CLas influye sobre la dinámica de población de microorganismos presentantes en la rizosfera de plantas de limón, observando solo los resultados para los tratamientos QCLas y Q, se muestra que al comparar el tratamiento infectado (QCLas) con el tratamiento sin infectar (Q), la dinámica poblacional de bacterias totales es modificada (Cuadro 2). A 70 ddpa, el conteo de UFC entre el tratamiento Q y QCLas no muestra diferencias estadísticas (Tukey, $P \leq 0.05$ ) entre sí, pero si a los tratamientos inoculados con microrganismos benéficos (Cuadro 2). Los tratamientos Q y QCLas a 140,210, 280 y 368 ddpa muestran diferencias estadísticas (Tukey, $P \leq 0.05$ ) entre sí, siendo para el tratamiento Q el conteo de UFC mayor en tres de cuatro muestreos (Cuadro 2). Además, en 3 de los 4 muestreos el conteo de UFC fue mayor en el tratamiento Q. A partir de 210 ddpa el conteo de UFC g-1 de sustrato para el tratamiento QCLas disminuyó, en contraste al tratamiento Q que incluso el conteo de UFC va en aumento. Indicando que, posiblemente $C$ Las inhibe el crecimiento de microorganismos presentes en la rizósfera, aunque, se debe tener en cuenta que CLas es un fitopatógeno que se aloja en el floema de los cítricos. A 368 ddpa el tratamiento Q mostró el mayor conteo de UFC con respecto a los tres tratamientos infectados con CLas (Cuadro 2). Hacquard et al. (2017) mencionan que organismos patógenos adaptados a sus hospederos pueden manipular el sistema inmune de la planta para su propio beneficio, pero también en formas que tienen consecuencias para otros microorganismos. Agler et al. (2016), observaron que el patógeno oomiceto perteneciente al género Albugo, tiene efectos en la colonización de bacterias epifitas y endófitas, disminuyendo la diversidad de las $\alpha$-bacterias y estabilizando la diversidad de las $\beta$-bacterias. Por otra parte, Wang et al. (2017) mencionan que $C$ Las causa cambios dramáticos en el metabolismo y el sistema regulatorio de los cítricos, afectando la composición de los exudados radicales e indirectamente la microbiota de la rizósfera. CLas también afecta el sistema de defensa de los cítricos que en consecuencia impacta en la microbiota de la rizósfera (Wang y Trivedi, 2013). La abundancia del microbioma de la rizósfera de plantas de cítricos infectadas con CLas es menor en comparación con el de plantas de cítricos sanas, así mismo se ve afectado la composición taxonómica disminuyendo la abundancia de los géneros Burkholderia y Pseudomonas y aumenta la proporción de Acidobacteria, Actinobacteria, Firmicutes y Bacillus (Trivedi et al., 2012).

En contraste, el conteo de hongos filamentosos totales (Cuadro 3) que solamente en el muestreo realizado a 140 ddpa se observó un conteo mayor en las UFC g $\mathrm{g}^{-1}$ de sustrato correspondiente al tratamiento QCLas con respecto al tratamiento Q. Pudiendo decir que la infección de limón con CLas, no induce cambios en la dinámica de población en la rizósfera de hongos filamentosos. Cabe resaltar que el conteo de hongos filamentosos aumentó a 368 ddpa con respecto a los muestreos anteriores, efecto contrario a la población de bacterias totales, la cual va decreciendo con el tiempo.

\section{Influencia de la Nutrición Orgánica en las Poblaciones de Bacterias y Hongos Filamentosos Totales en Plantas de Limón Infectadas con CLas}

Con el fin de evaluar el efecto de la nutrición orgánica en la dinámica de microorganismos de 
la rizósfera en limón infectado con CLas se incluyeron dos tratamientos a los que se les varió la nutrición de química a orgánica. Los resultados demostraron que para el caso de las bacterias totales a 71 ddpa no existe diferencia estadística (Tukey, $P \leq 0.05$ ) entre los tratamientos (Figura 2).A140 ddpa el conteo de bacterias totales aumentó significativamente llegando a tener hasta $1.35 \times 10^{7} \mathrm{UFC} \mathrm{g}^{-1}$ de sustrato para el tratamiento QCLas y un mínimo de $2.57 \times 10^{6} \mathrm{UFC} \mathrm{g}^{-1}$ de sustrato para el tratamiento $\mathrm{O}$ (Figura 2). No obstante, a partir de 210 ddpa se aprecia un decremento en el conteo de UFC de bacterias totales, teniendo a 368 ddpa un conteo máximo de $2.67 \times 10^{6} \mathrm{UFC} \mathrm{g}^{-1}$ de sustrato para el tratamiento Q (Figura 2). En el conteo de UFC de los hongos filamentosos totales se observa un decremento a 70 ddpa en los tratamientos que recibieron nutrición orgánica y un aumento en las UFC en los tratamientos que recibieron nutrición química en comparación con las UFC del sustrato inicial; sin embargo, no mostraron diferencias significativas (Tukey, $P \leq 0.05$ ) entre ellos (Figura 3). El comportamiento de la dinámica de población de los hongos filamentosos totales con nutrición orgánica se puede considerar estable, ya que su población se mantuvo entre 1000 y $2000 \mathrm{UFC} \mathrm{g}^{-1}$ de sustrato (Figura 3). En contraste con los tratamientos con nutrición química cuya población fue de $1000 \mathrm{UFC} \mathrm{g}^{-1}$ de sustrato a un poco más de $5000 \mathrm{UFC} \mathrm{g}^{-1}$ de sustrato a 368 ddpa, mostrando una tendencia al aumento de la población de hongos filamentosos tanto para el tratamiento QCLas y Q (Figura 3).

Las poblaciones de bacterias se mantuvieron en un intervalo de $10^{5}$ a $10^{6} \mathrm{UFC} \mathrm{g}^{-1}$ de sustrato (Figura 2). Siendo reportado por Calvo et al. (2008) un conteo de bacterias totales en un intervalo de $10^{7}$ y $10^{8} \mathrm{UFC} \mathrm{g}^{-1}$ de sustrato en muestras de rizósfera de cultivo de papa. Bolaños et al. (2013) reportaron conteos de bacterias totales de $10^{6} \mathrm{UFC}^{-1}$ de sustrato en muestras de rizósfera de palma aceitera (Elaeis guineensis Jacq) con riego y sin riego, cultivada en campo abierto. En cuanto a los hongos filamentosos estos se mantuvieron en un intervalo de $10^{3} \mathrm{UFC}^{-1}$ de sustrato (Figura 3). Calvo et al. (2008) reportaron conteos de hongos filamentosos en un intervalo de $10^{4}$ a $10^{5} \mathrm{UFC} \mathrm{g}^{-1}$ de sustrato. Bolaños et al. (2013) reportaron conteos de hongos totales de $10^{3} \mathrm{UFC} \mathrm{g}^{-1}$ de sustrato en parcelas con riego y de $10^{4} \mathrm{UFC} \mathrm{g}^{-1}$ de sustrato en parcelas sin riego. En general se observa una disminución de la carga bacteriana total en los tratamientos de plantas

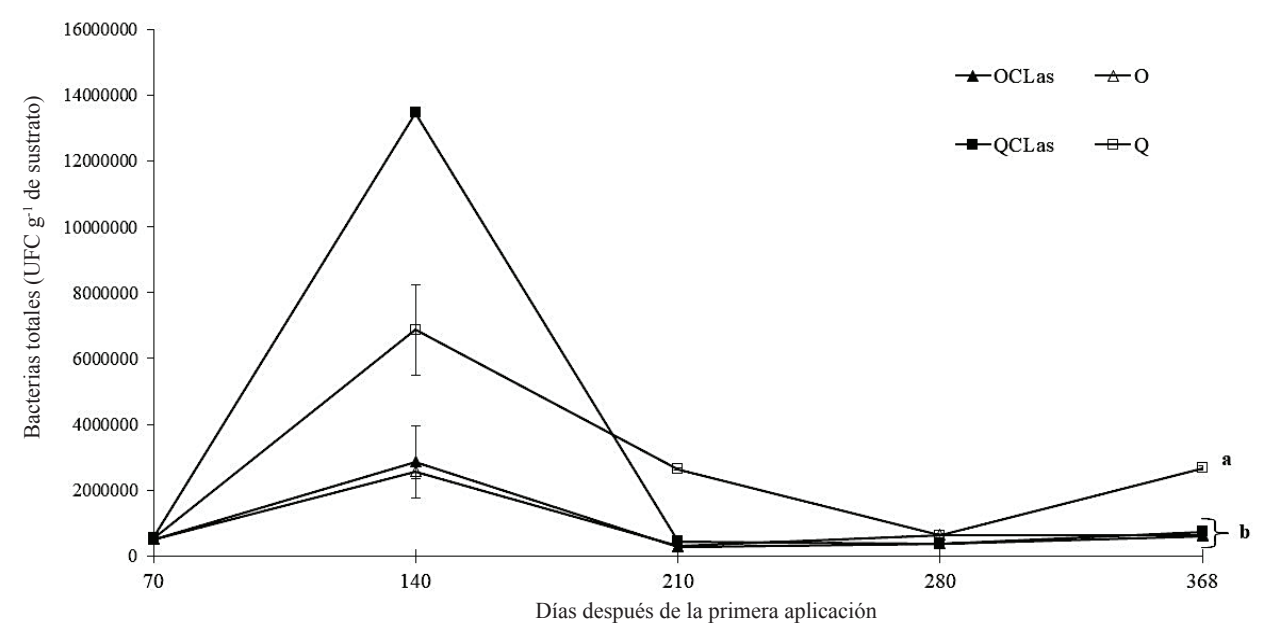

Figura 2. Dinámica de las poblaciones bacterianas totales después de la primera aplicación de nutrición orgánica en árboles de limón mexicano bajo condiciones de invernadero. Fertilización orgánica (O), química (Q), CLas = inoculación con Candidatus Liberibacter asiaticus. Las barras en cada punto de muestreo indican \pm error estándar. Letras distintas al final del experimento indican diferencias significativas, según la prueba de Tukey $(P \leq 0.05)$.

Figure 2. Population dynamics of total bacteria after the first application of organic nutrition in Mexican lime trees under greenhouse conditions. Organic fertilization (O), chemical (Q), CLas = inoculation with Candidatus Liberibacter asiaticus. Bars in each sampling point indicate \pm standard error. Different letters at the end of the experiment indicate significant differences, according to the Tukey's test $(P \leq 0.05)$. 


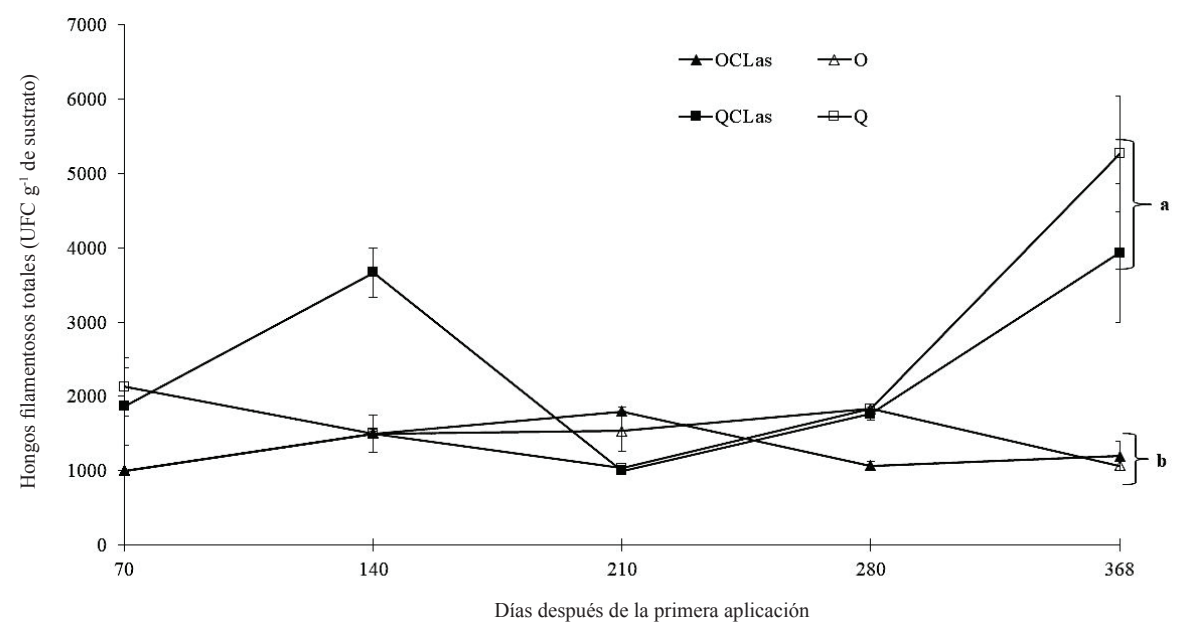

Figura 3. Dinámica de poblaciones de hongos filamentosos totales después de la primera aplicación de nutrición orgánica en árboles de limón mexicano bajo condiciones de invernadero. Fertilización orgánica $(\mathrm{O})$, química $(\mathrm{Q})$, CLas = inoculación con Candidatus Liberibacter asiaticus. Las barras en cada punto de muestreo indican \pm error estándar. Letras distintas al final del experimento indican diferencias significativas, según la prueba de Tukey $(P \leq 0.05)$.

Figure 3. Population dynamics of total filamentous fungi after the first application of organic nutrition in Mexican lime trees under greenhouse conditions. Organic fertilization $(\mathrm{O})$, chemical (Q), CLas = inoculation with Candidatus Liberibacter asiaticus. Bars in each sampling point indicate \pm standard error. Different letters at the end of the experiment indicate significant differences, according to the Tukey's test $(P \leq 0.05)$.

de limón infectadas con CLas. Sin embargo, esta disminución fue notoria también para las plantas sanas, es decir, para aquellas que no fueron infectadas. El fenómeno anterior se puede justificar debido a la alta competencia entre los microorganismos que se desarrolla en la rizósfera por adquirir los nutrimentos encontrados en los exudados radicales (Yagüe y Gutiérrez-Correa, 1982). Además, los exudados radicales fungen como atrayentes y repelentes químicos en la rizósfera y su zona de influencia regulando las comunidades microbianas del suelo (Torres-Guerrero et al., 2013). Lo que deja abierto el análisis de los exudados radicales en plantas de limón infectadas con CLas y su influencia en la dinámica poblacional bacterias y hongos filamentosos. El crecimiento en la población de microorganismos en el suelo puede estar influenciado por varios factores desde la materia orgánica disponible para su crecimiento hasta cambios de $\mathrm{pH}$ por cuestiones biológicas y bioquímicas. Sin embargo, la inoculación con los microorganismos benéficos como Funneliformis mosseae y Bacillus subtilis no aumentaron las poblaciones de bacterias totales. Sino al contrario, estas disminuían conforme avanzaba la enfermedad.

\section{CONCLUSIONES}

Con el comportamiento observado entre las poblaciones de bacterias y hongos filamentos totales, en muestras de sustrato de plantas de limón mexicano infectadas con Candidatus Liberibacter asiaticus (Clas), se encontró que la aplicación de microorganismos benéficos como Funneliformis mosseae y Bacillus subtilis aumentan las poblaciones de bacterias y hongos filamentosos totales en plantas infectadas con CLas y las mantienen estables hasta 210 días después de iniciado el experimento. La aplicación de nutrición orgánica no influye en la dinámica de población de bacterias totales en plantas de limón mexicano infectadas con CLas; en cambio, la aplicación de nutrición orgánica mantiene estables las poblaciones de hongos filamentosos a lo largo del tiempo tanto en el tratamiento infectado como el tratamiento sin CLas. La aplicación de microorganismos benéficos y la nutrición orgánica hacen sinergia en la estabilización de hongos filamentosos totales en plantas de limón mexicano infectadas con CLas. Se encontraron diferencias estadísticas en el tratamiento infectado con CLas y que recibió nutrición química en comparación 
con el tratamiento sin infectar, lo que demuestra que la infección con CLas en plantas de limón influye negativamente en el desarrollo poblacional de bacterias totales presentes en la rizósfera.

\section{AGRADECIMIENTOS}

Se agradece al proyecto: Efecto de distintos bioprotectores en el desarrollo de HLB en limón mexicano del estado de Michoacán: 2012-03-193066 de Fondo Mixto de Fomento Científico y Tecnológico CONACYT-Gobierno del Estado de Michoacán. La Dra. Evangelina Esmeralda Quiñones-Aguilar fue codirectora de la tesis de Christian Salvador Mendoza Hernández.

\section{LITERATURA CITADA}

Agler, M., J. Ruhe, S. Kroll, C. Morhenn, S. Kim, D. Weigel, and E. Kemen. 2016. Microbial hub taxa link host and abiotic factors to plant microbiome variation. PloS Biol 14: 1-31. doi: http:// doi.org/10.1371/journal.pbio.1002352.

Andrade-Torres, A. 2010. Micorrizas: antigua interacción entre plantas y hongos. Ciencia 61: 84-90.

Armenta-Cárdenas, I., J. E. Ortiz-Enríquez, A. Vega-Verdugo y J. M. Ramírez-Díaz. 2010. Presencia de Diaphorina citri en el valle de Guaymas-Empalme, Sonora. 2007-2010. pp. 8-12. In: CONACYT-SAGARPA. $1^{\text {er }}$ Simposio Nacional sobre investigación para el manejo del psílido asiático de los cítricos y el huanglongbing en México-2010. Monterrey, Nuevo León, México. https://sites.google.com/site/diaphorina/ simposiohlb1. (Consulta: enero 30, 2019).

Bolaños, C., M. Estrada, H. Orellana y G. Bernal. 2013. Influencia del riego en la dinámica poblacional de grupos funcionales de microorganismos asociados a la rizósfera de tres materiales tenera de palma aceitera (Elaeis guineensis Jacq.). pp. 73-80. In: Asociación Nacional de Cultivadores de Palma Africana, Ancupa (eds.). Investigaciones en palma aceitera: Recopilación de estudios, conocimientos y productividad desarrollados por el CIPAL. ANCUPA. Ecuador. doi: http://doi.org/10.13140/ RG.2.1.1972.9360.

Calvo V., P., L. R. Meneses y D. Zúñiga D. 2008. Estudio de las poblaciones microbianas de la rizósfera del cultivo de papa (Solanum tuberosum) en zonas altoandinas. Ecol. Aplic. 7: 141-148. doi: http://dx.doi.org/10.21704/rea.v7i1-2.369.

Camacho, A., M. Giles, A. Ortegón, M. Palao, B. Serrano y O. Velázquez. 2009. Técnicas para el análisis microbiológico de alimentos. Facultad de Química, UNAM. México, D. F.

Camargo-Ricalde, S., N. M. Montaño, C. J. de la Rosa-Mera y S. A. Montaño Arias. 2012. Micorrizas: Una gran unión debajo del suelo. Rev. Digital Univ. 13: 1-19. http://www.revista.unam. $\mathrm{mx} /$ vol.13/num7/art72/index.html.

Cui, J. Q., H. B. Sun, M. B. Sun, R. T. Liang, W. G. Jie, and B. Y. Cai. 2018. Effects of Funneliformis mosseae on root metabolites and rhizosphere soil properties to continuouslycropped soybean in th potted-experiments. Int. J. Mol. Sci. 19: E2160. doi: 10.3390/ijms19082160.
De-Bashan, L. E., G. Holguin, B. R. Glick y Y. Bashan. 2007. Bacterias promotoras de crecimiento en plantas para propósitos agrícolas y ambientales. pp. 161-215. In: R. Ferrera-Cerrato y A. Alarcón. (ed.). Microbiología agrícola: Hongos, bacterias, micro y macrofauna. Control biológico y plantamicroorganismo. Trillas. México, D. F. ISBN: 9789682478109 9682478103.

González F., H. y N. Fuentes. 2017. Mecanismo de acción de cinco microorganismos promotores de crecimiento vegetal. Revista de Cienc. Agríc. 34: 17-31. doi: http://dx.doi.org/10.22267/ rcia.173401.60.

González-Sánchez, R. F. y R. S. Echevarria. 2003. Caracterización de las cadenas prioritarias e identificación de las demandas tecnológicas: cadena de limón mexicano (Citrus aurantifolia Swingle). Coordinación general de vinculación dirección general de estudios estratégicos y la Facultad de Economía, Colima. (eds.). Programa estratégico de investigación y transferencia de tecnología en el estado de Colima. Universidad de Colima y Fundación Produce Colima, A.C. Colima, México. http://siic.ucol.mx/Archivos_prov\%5CDiagnostico\%20 Limon.pdf. (Consulta: marzo 15, 2019).

Gui, H., W. Purahong, K. D. Hyde, J. Xu, and P. E. Mortimer. 2017. The arbuscular mycorrhizal fungus Funneliformis mosseae alters bacterial communities in subtropical forest soils during litter decomposition. Front. Microbiol. 8: 1120. doi: https:// doi.org/10.3389/fmicb.2017.01120.

Hacquard, S., S. Spaepen, R. Garrido-Oter, and P. SchulzeLefert. 2017. Interplay between innate immunity and the plant microbiota. Annu. Rev. Phytopathol. 55: 565-589. doi: 10.1146/annurev-phyto-080516-035623.

Hernández-Cuevas, L., S. Castillo-Argüero, P. GuadarramaChávez, Y. Martínez-Orea, M. A. Romero-Romero e I. Sánchez-Gallén. 2003. Hongos micorrizógenos arbusculares del Pedregal de San Ángel. UNAM. México, D.F. ISBN: $9703204317,9789703204311$.

Jagoueix, S., J. M. Bove, and M. Garnier. 1994. The phloemlimited bacterium of greening disease of citrus is a member of the $\alpha$ subdivision of the Proteobacteria. Int. J. Syst. Bacteriol. 44: 379-86.

Kim, J. S., U. S. Sagaram, J. K. Burns, J. L. Li, and N. Wang. 2008. Response of sweet orange (Citrus sinensis) to "Candidatus Liberibacter asiaticus" infection: Microscopy and microarray analyses. Phytopathology 99: 50-57. doi: 10.1094/ PHYTO-99-1-0050.

Loredo-Osti, C., L. López-Reyes y D. Espinosa-Victoria. 2004. Bacterias promotoras del crecimiento vegetal asociadas con gramíneas: Una revisión. Terra Latinoamericana 22: 225-239.

Lou, B. H, X. L. Zhao, Y. Q. Song, X. J. Bai, C. L. Deng, and G. P. Chen. 2012. "Candidatus Liberibacter asiaticus", associated with citrus huanglongbing, infects pollen, seed coat and endosperm of pummelo in China. J. Plant Pathol. 93: 703-705. doi: http://dx.doi.org/10.4454/JPP.FA.2012.072.

NOM-021-RECNAT-2000 (Norma Oficial Mexicana). 2002. Que establece las especificaciones de fertilidad, salinidad y clasificación de suelos. Estudios, muestreo y análisis. SEMARNAT. México, D. F. http://diariooficial.gob.mx/nota detalle.php? codigo $=717582 \&$ fecha $=31 / 12 / 2002$ (Consulta: marzo 10, 2019).

Ramírez Gil, J. G., D. A. Castañeda-Sánchez y J. G. MoralesOsorio. 2013. Dinámica microbial del suelo asociada a diferentes estrategias de manejo de Phytophthora cinnamommi Rands en aguacate. Rev. Ceres 60: 811-819. doi: http://dx.doi. org/10.1590/S0034-737X2013000600009. 
Robles-González, M. M., J. J. Velázquez-Monreal, M. A. Manzanilla-Ramírez, M. Orozco-Santos, R. Flores-Virgen y V. M. Medina-Urrutia. 2010. Síntomas del huanglongbing en limón mexicano. Primeras observaciones. 021 068. In: $1^{\text {er }}$ Simposio Nacional sobre investigación para el manejo del psílido asiático de los cítricos y el huanglongbing en México-2010 CONACYT-SAGARPA. Monterrey, Nuevo León, México. https://sites.google.com/site/diaphorina/ simposiohlb1. (Consulta: enero 30, 2019).

Sánchez-Borja, M., H. Bravo-Mojica, J. Romero-Nápoles, S. Ramírez-Alarcón y B. López-Martínez. 2010. Biología, ecología y control de Diaphorina citri Kuwayama (Hemiptera Psyllidae) 006 026. In: $1^{\text {er }}$ Simposio Nacional sobre investigación para el manejo del psílido asiático de los cítricos y el huanglongbing en México-2010 CONACYT-SAGARPA. Monterrey, Nuevo León, México. https://sites.google.com/ site/diaphorina/simposiohlb1. (Consulta: enero 30, 2019).

Santillano-Cázares, J., L. G. Roque-Díaz, F. Núñez-Ramírez, R. L. Grijalva-Contreras, F. Robles-Contreras, R. Macías-Duarte, I. Escobosa-García y V. Cárdenas-Salazar. 2019. La fertilidad del suelo afecta el crecimiento, nutrición y rendimiento de algodón cultivado en dos sistemas de riego y diferentes dosis de nitrógeno. Terra Latinoamericana 37: 7-14. doi: https://doi. org/10.28940/terra.v37i1.304.

Sarabia-Ochoa, M., R. Madrigal-Pedraza, M. Martínez-Trujillo y Y. Carreón-Abud. 2010. Plantas, hongos micorrízicos y bacterias: su compleja red de interacciones. Biológicas 12: 65-71.

Shen, W., J. M. Cevallos-Cevallos, U. Nunes da Rocha, H. A. Arevalo, P. A. Stansly, P. D. Roberts, and A. H. C. van Bruggen. 2013. Relation between plant nutrition, hormones, insecticide applications, bacterial endophytes, and Candidatus Liberibacter $\mathrm{Ct}$ values in citrus trees infected with Huanglongbing. Eur. J. Plant Pathol. 137: 727-742. doi: 10.1007/s10658-013-0283-7.

SIAP (Servicio de Información Agroalimentaria y Pesquera). 2019. Avance de siembras y cosechas: Resumen nacional por cultivo. Obtenido de Reportes: http://infosiap.siap.gob. mx:8080/agricola_siap_gobmx/AvanceNacionalSinPrograma. do. (Consulta: enero 30, 2019).
StatPoint Inc. 2005. StatGraphics Centurion XV version 15.02.06. Warrenton, VA, USA. www.statgraphics.com

Torres-Guerrero, C., J. D. Etchevers B., M. H. Fuentes-Ponce, B. Govaerts, F. de León-González y J. M. Herrera. 2013. Influencia de las raíces sobre la agregación del suelo. Terra Latinoamericana 31: 71-84.

Trinidad-Cruz, J. R., E. E. Quiñones-Aguilar, G. RincónEnríquez, L. López-Pérez y L. V. Hernández-Cuevas. 2017. Mycorrhization of Agave cupreata: biocontrol of Fusarium oxysporum and plant growth promotion. Rev. Mex. Fitopatol. 35: 151-169. doi: 10.18781/R.MEX.FIT.1607-5.

Trivedi, P., Z. He, J. D. Van Nostrand, G. Albrigo, J. Zhou, and N. Wang. 2012. Huanglongbing alters the structure and functional diversity of microbial communities associated with citrus rhizosphere. ISME J. 6: 363-383. doi: 10.1038/ ISMEJ.2011.100.

Wang, N. and P. Trivedi. 2013. Citrus huanglongbing: A newly relevant disease presents unprecedented challenges. Phytopathology 103: 652-665. doi: 10.1094/PHYTO-12-120331-RVW.

Wang, N., L. L. Stelinski, K. S. Pelz-Stelinski, J. H. Granham, and Y. Zhang. 2017. Tale of the huanglongbing disease pyramid in the context of the citrus microbiome. Phytopathology 107: 380-387. doi: 10.1094/PHYTO-12-16-0426-RVW.

Wang, X. Q., D. L. Zhao, L. L. Shen, C. L. Jing, and C. S. Zhang. 2018. Application and mechanisms of Bacillus subtilis in biological control of plant disease. pp. 225-250. In: V. S. Meena (ed.). Role of rhizospheric microbes in soil: Volume 1: Stress management and agricultural sustainability. Springer. New York, NY, USA. doi: 10.1007/978-981-10-8402-7_9.

Yagüe, N. y M. Gutiérrez-Correa. 1982. Dinámica poblacional de las bacterias del ciclo del nitrógeno en los suelos de las lomas de Lachay. Zonas Áridas 1: 75-80.

Zhang, Y. P., J. K. Uyemoto, and B. C. Kirkpatrick. 1998. A smallscale procedure for extracting nucleic acids from woody plants infected with various phytopathogens for PCR assay. J. Virol. Methods 71: 45-50. 